\title{
Research and Implementation of Network Trouble - Shooting Training Project
}

\author{
Qiang Miao' ${ }^{1}$, Hui Li ${ }^{2}$, and Hong Wang ${ }^{3}$ \\ ${ }^{1}$ Department of Computer Science and Technology, Dalian Neusoft University of Information, Dalian, \\ Liaoning, 116023; \\ ${ }^{2}$ Department of Electronic Engineering, Dalian Neusoft University of Information, Dalian, Liaoning, \\ 116023; \\ ${ }^{3}$ Department of Computer Science and Technology, Dalian Neusoft University of Information, Dalian, \\ Liaoning, 116023
}

\begin{abstract}
In order to cultivate the application type of network engineering professionals, make students familiar with the network principles and solve the issue of insufficient ability to eliminate network failures, the software design based on Cisco Packet Tracer realizes the price of network failure trouble shooting combines theories with practices. This paper analyzes the knowledge points in professional network engineering course and technical points in network construction and maintenance, and designs and makes corresponding network troubleshooting training projects with the Activity Wizard function of PT software. The training project file has automatic scoring function and troubleshooting guidance function. The project system can be extended to classroom teaching and practical teaching, which can improve students' hands-on ability in the network troubleshooting.
\end{abstract}

Keywords: Network trouble; Packet tracer; Network engineering; Practical teaching

\section{网络故障排除训练项目的研究与实现}

\author{
苗强 ${ }^{1}$ ，李慧 ${ }^{2}$, 王红 ${ }^{3}$
}

(1. 大连东软信息学院 计算机科学与技术系, 辽宁 大连 $116023 ; 2$. 大连东软信息学院电子工程系, 辽 宁 大连 116023；3. 大连东软信息学院 计算机科学与技术系, 辽宁 大连 116023)

摘要: 为了培养应用型的网络工程专业人才, 解决学生熟悉网络原理, 但是排除网络故障的能力有所欠缺的问题。基于 Cisco Packet Tracer 软件设计实现了网络故障排除训练项目。将理论与实践相结合, 分析网络工程专业课程的知识点和网络 组建和维护工作中涉及的技术点, 借助 PT 软件的 Activity Wizard 功能设计和制作相应的网络故障排除训练项目。训练项目 文件具有自动评分功能和排错引导功能。将项目体系推广到课堂教学和实践教学中, 可以提高学生在网络故障排除中的动手 能力。

关键词: 网络故障; Packet Tracer; 网络工程; 实践教学

中图分类号: TP 393 文献标志码: A

引言

很多高校的网络工程专业或者网络技术专业, 都开设了网络组建和维护类的课程, 比如《网络互联技 术》、《组网技术》和《路由与交换技术》等。目标是培养学生使用路由器、交换机等网络设备组建网络的 能力。这类课程重在实践能力的培养和提升。但是如今网络工程专业的学生熟悉网络原理, 但是排除网络 故障的能力有所欠缺。为了培养应用型的网络工程专业人才, 设计开发基于 Packet Tracer 的网络故障排 除训练项目。项目将理论与实践相结合, 分析网络工程各个专业课程的知识点, 制作相应的网络故障排除 
训练项目。并且将这个项目应用于课堂教学和实践教学, 培养学生在网络故障排除中的实际动手能力。

\section{Packet Tracer 软件介绍和使用}

\section{1 Cisco Packet Tracer 介绍}

Cisco Packet Tracer 是由 Cisco 公司开发的一个开源的辅助学习软件 ${ }^{[1]}$ 。为学习网络类课程的学生, 在设计、组建和配置网络过程中提供了网络模拟环境。使用者可以在软件的图形化界面上直接使用拖电方 式建立网络拓扑, 并可以根据数据包在网络中传输的详细过程, 观察网络实时运行情况。软件对网络设备 的模拟做的很好, 可以同时模拟几十台网络设备的运行, 很好的解决了网络组建模拟实验中, 网络实验设 备数量有限的问题。

\section{2 基于 Packet Tracer 的 pkt 和 pka 文件的制作}

项目是使用 Cisco Packet Tracer 来实现的 ${ }^{[2]}$ 。第一步, 针对网络组建和维护中涉及的知识点, 创建 一个已经搭建好网络拓扑的. pkt 文件 (正确版)。第二步, 在之前正确版的基础上, 根据预先设定的网络 故障加入故障点的配置, 制作. pkt 文件 (错误版)。第三步, 组合第一步和第二步的文件, 形成基于网络 故障排除的多个.pka 文件 (排错版)。

在制作正确版.pkt 文件的时候, 首先要设定一个合理的虚拟的网络拓扑的背景。比如 “WCG 股份有限 公司是一家新成立的 IT 企业, 企业规模为 100 人, 财务部、行政部、业务部、管理部和后勤部为公司 5 个基本部门”。还要对已有的网络拓扑和配置做简单的描述。比如 “公司总部所有路由器上运行 OSPF 协议, 进程号为 110 。路由器 $R 1$ 的 $f 0 / 0$ 和 $f 0 / 1$ 运行在 area 0 内” 等等。

在制作错误版. pkt 文件的时候, 需要根据网络维护的经验预先设定故障点。比如设置 OSPF 协议的故 障点, 修改 OSPF 协议的进程号为 100 , 这样就会导致通信失败, 就可以要求学生通过查看现象或者配置文 件发现这个故障点并排除。

最后需要使用 Packet Tracer 的 Activity Wizard 功能将第一步的正确版的. pkt 文件和第二步的错误 版的. pkt 文件组合在一起, 制作具有评分功能和排错引导功能的. pka 文件, 即项目最终呈现给学生的排错 版项目文件 ${ }^{[3]}$ 。而. pka 文件的制作也要注意几个环节。首先需要使用到 “Instructions” 模块, 将网络拓 扑背景, 网络环境的描述介绍清楚 ${ }^{[4][5]}$, 如图 1 所示。其次使用 “Initial Network” 模块, 将错误版的. pkt

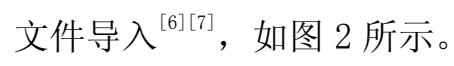

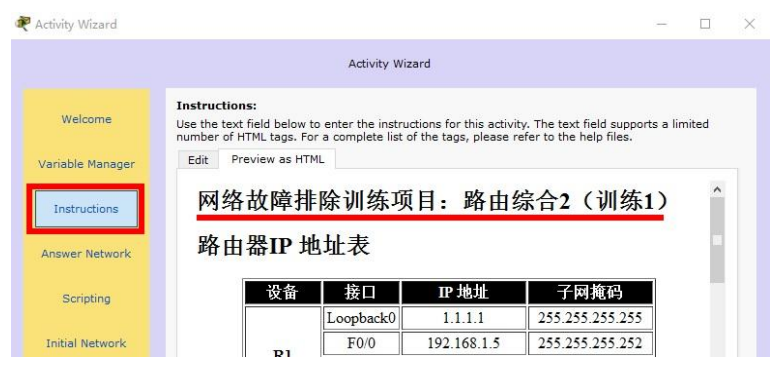

图 1 Instructions 模块

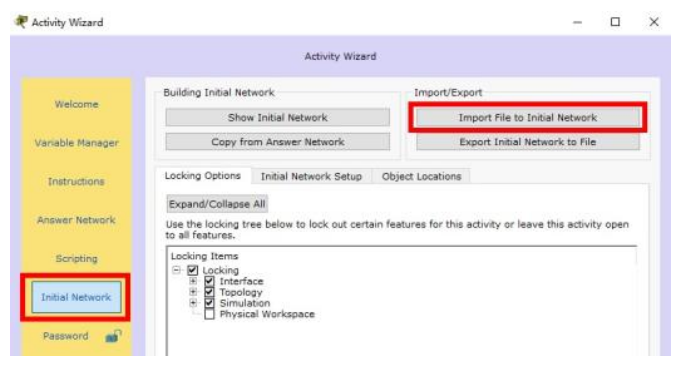

图 2 Initial Network 模块

最后使用 “Answer Network” 模块, 将正确版的. pkt 文件导入, 如图 3 所示, 并且正确的设置给分点。 使得学生在正确的找到故障点并排除故障的时候, 系统自动识别评分 [8]。 


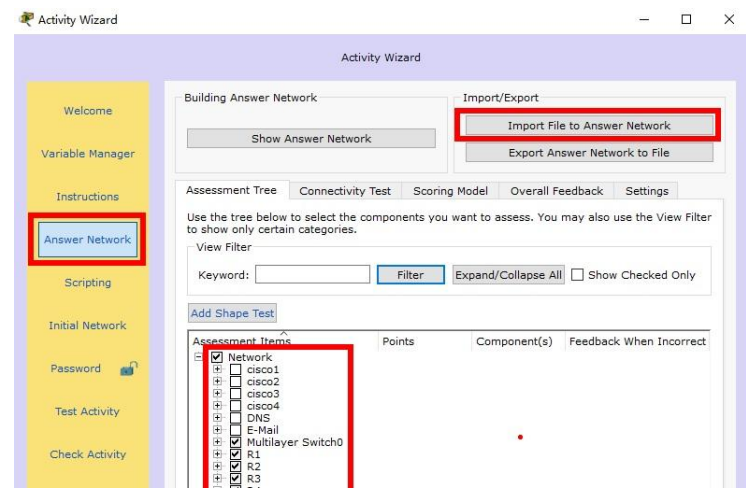

图 3 Answer Network 模块

\section{2 网络故障排除训练项目的实现}

\section{1 项目的设置}

故障排除训练项目在知识体系上, 涵盖了网络工程专业学生所学习的网络组建类的专业课程。系统的 整理和分析网络知识点, 保证整理的知识点的正确性和全面性。同时根据学习和教学中总结的经验, 挖掘 通信过程中容易出现的故障点, 和网络搭建过程中容易出现的错误。针对不同的知识点设置了若干个排错 项目，项目列表如表 1 所示。

表 1 项目列表

\begin{tabular}{ll|ll|ll|ll}
\hline 序号 & 项目名称 & 序号 & 项目名称 & 序号 & 项目名称 & 序号 & 项目名称 \\
\hline 1 & IOS & 2 & IP 地址规划 & 3 & vlan & 4 & VTP \\
5 & 链路聚合 & 6 & HSRP & 7 & 交换综合 1 & 8 & 交换综合 2 \\
9 & 静态路由 & 10 & RIP & 11 & EIGRP 初级 & 12 & EIGRP 高级 \\
13 & OSPF 初级 & 14 & OSPF 高级 & 15 & 路由综合 1 & 16 & 路由综合 2 \\
17 & PPP 协议 & 18 & 帧中继 & 19 & ACL & 20 & NAT \\
21 & 安全 & 22 & 路由重发布 & 23 & BGP & 24 & 综合 1 \\
25 & 综合 2 & 26 & 综合 3 & 27 & 综合 4 & 28 & 综合 5 \\
\hline
\end{tabular}

\section{2 故障点的设置}

在某个具体的排错项目中，需要根据不同的知识点，不同的错误类型，部署相应的故障点。同时针对 一个排错项目的网络拓扑, 应该设置两个版本以上的错误版。在不同的错误版中设置不同的故障点。比如 IP 地址配置错误, 端口带宽、速率配置错误, 端口状态错误等。以综合 2 (排错版 1) 中的部分故障点设 置为例, 如表 2 所示。 
表 2 故障点设置表

\begin{tabular}{l|l|l}
\hline 设备名称 & 故障类型 & 故障点 \\
\hline \multirow{2}{*}{ R1 } & 接口 IP 地址错误 & 修改 F0/1 接口 IP 地址 \\
\cline { 2 - 3 } & 附加错误配置 & F1/0 接口带宽 \\
\hline \multirow{2}{*}{ R2 } & Router-id 重复 & 修改 OSPF router-id 与 R1 一致 \\
\cline { 2 - 3 } & 漏宣告网段 & 删除 192.168.1.8/30 网段 \\
\hline \multirow{2}{*}{ R3 } & 接口状态 & F0/1shutdown \\
\cline { 2 - 3 } & 附加错误配置 & 添加 DHCP pool cisco \\
\hline
\end{tabular}

\section{3 故障排除的问题引导}

在引导学生使用故障排除训练项目, 完成练习的过程中还需要给出明确的任务需求。比如 “财务部的 个人电脑 ciscol 无法和业务部的 cisco2 进行通信”。即将通信故障的现象通过文字描述呈现出来, 进而 引导学生发现故障点, 并能排除故障点。因为在真实的网络维护的工作中, 就是由用户反映网络通信出现 问题。而问题的故障原因是由网络管理人员通过专业知识逐一排查, 才能最终解决。所以通信故障引导的 好处就是, 贴近真实的工作流程。比如综合 2（排错版 1) 中的引导 “网络管理员接到来自各部门同事打 来的电话, 据他们反映无法正常与其他部门进行通讯, 也无法正常接收和发送邮件, 无法访问远程数据中 心的Web 服务”。

\section{3 网络故障排除训练项目的应用}

故障排除训练项目, 最终呈现给学生的是若干个. pka 文件。学生运行. pka 文件, 使用训练项目完成 练习的时候, 需要完成 “百分比任务” 和 “连通性测试任务” [9] [10], 如图 4 所示。通过命令查看路由 器 R1 的 F1/0 接口。发现配置 helper-address 错误, 使用命令 “ip helper-address 192.168.4.4” 将其 修改后百分比增加, 如图 5 所示。

\section{interface FastEthernet1/0 \\ ip address 192.168.5.1 255.255.255.192 \\ ip helper-address 192.168.4.3 \\ duplex auto \\ speed auto}

图 $4 \mathrm{R} 1$ 的 $\mathrm{F} 1 / 0$ 接口配置图

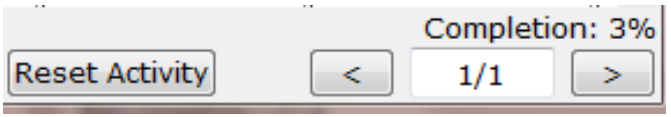

图 5 故障排除后的完成百分比的增加

练习过程中正确的找到全部的故障点并进行排除，同时保证任务中要求的主机之间的连通性成功，即 通信测试列表中显示 “successful”, 则自动评分功能的完成百分比会达到 $100 \%$ ，代表故障点都被排除了。

\section{结语}

网络故障排除训练项目, 已经应用于 12 级--15 级网络工程专业和网络技术学生的实践教学环节中。 通过观察和统计可以发现, 项目的应用已经取得了一定的成果, 切实的提高了学生的网络故障排除的能力。

\section{致谢}

辽宁省普通高等学校本科工程人才培养模式改革试点专业项目 
大连东软信息学院创新创业教育试点专业项目

\section{Acknowledgement}

Liaoning Provincial Higher Education Undergraduate Engineering Training Model Reform Pilot Project

Dalian Neusoft University of Information Innovation Entrepreneurship Education Pilot Project

参考文献

[1] 谭艈银, 王平. PacketTracer 软件在计算机网络实验教学中的应用 [J]. 软件导刊, 2015，14(4)：169-170.

[2] 苏新, 刘俞, 毛万胜. PacketTracer 在网络组建类课程考核中的应用 [J]. 辽东学院学报, 2012, 19 (4) : 273-276.

[3] 马国峰，杨俊红．仿真软件在网络教学及考核中的应用[J]. 济源职业技术学院学报，2016，15(3)：87-91.

[4] 王海风, 刘志强. 基于 PacketTracer 的计算机网络类课程实验测评系统的研究与实现 [J]. 内蒙古工业大学学报, 2015, 34(2): 125-130.

[5] 王为群．基于 PT 的实验考核系统设计与实现 [J]. 韶关学院学报, 2016，37(4)：13-17.

[6] 杨涁．基于 PacketTracer 环境下的网络课程考核平台构建 [J]. 计算机教育, 2010，2010(23)：143-146.

[7] 陈华. 基于 PacketTracer 的 Multiuser 扩展构建分布式网络模拟实验环境 $[\mathrm{J}]$. 计算机时代, 2011，2011(4)： $34-35$.

[8] 容振邦. 基于 PacketTracer 的计算机网络原理课程案例教学 [J]。计算机教育, 2011，2011(3)：67-70.

[9] 徐洪学, 郭秀英. 仿真软件 PacketTracer 在计算机网络工程课程教学中的应用 [J]. 沈阳教育学院学报, 2010, $12(1)$ : 84-88.

[10］支俊, 陈燕. 案例分析在本科软件工程教育中的应用研究 $[J]$. 软件导刊, 2010，14(7)：30-32.

\section{References}

[1] Tan Shuyin, Wang Pin. Application of PacketTracer in Computer Network Experiment Teaching [J]. Software Guide, 2015, 14(4): 169-170.

[2] Su Xin, Liu Yu, Mao Wansheng. Application of PacketTracer in the Course of Network Establishment [J]. Journal of Liaodong University, 2012, 19(4): 273-276.

[3] Ma Guofeng, Yang Junhong. Application of Simulation Software in Network Teaching and Examination [J]. Journal of Jiyuan Vocational and Technical College, 2016, 15(3): 87-91.

[4] Wang Haifeng, Liu Zhiqiang. Research and Implementation of Computer Network Course Experiment Evaluation System Based on PacketTracer [J]. Journal of Inner Mongolia University of Technology, 2015, 34(2): 125-130.

[5] Wang Weiqun. Design and Implementation of Examination System Based on PT [J]. Journal of Shaoguan University, 2016, 37(4): 13-17.

[6] Yang Bin. Construction of Network Course Evaluation Platform Based on PacketTracer [J]. Computer Education, 2010, 2010(23): 143-146.

[7] Chen Hua. Construction of Distributed Network Simulation Environment Based on Multiuser Extension of PacketTracer [J]. Computer Age, 2011, 2011(4): 34-35.

[8] Rong Zhenbang. Case Teaching of Computer Network Course Based on PacketTracer [J]. Computer Education, 2011, 2011(3): 67-70.

[9] Xu Hongxue, Guo Xiuying. The Application of Simulation Software PacketTracer in the Teaching of Computer Network Engineering [J]. Journal of Shenyang College of Education, 2010, 12(1): 84-88.

[10]Zhi Jun, Chen Yan. Application Research on Software Engineering Undergraduate Education in the Analysis of the Case [J]. Software Guide, 2010, 14(7): 30-32. 